\title{
Extreme slim accretion disks and narrow line Seyfert 1 galaxies: The nature of the soft X-ray hump
}

\author{
J.-M. Wang ${ }^{1,2,3}$ and H. Netzer ${ }^{1}$ \\ 1 School of Physics and Astronomy and the Wise Observatory, Tel Aviv University, Tel Aviv 69978, Israel \\ ${ }^{2}$ Laboratory of High Energy Astrophysics, Institute of High Energy Physics, Chinese Academy of Sciences, Beijing 100039, \\ PR China \\ ${ }^{3}$ Institut für Astronomie und Astrophysik, Abt. Astronomie, Universität Tübingen, Sand 1, 72076 Tübingen, Germany
}

Received 3 July 2002 / Accepted 10 October 2002

\begin{abstract}
We present a detailed model of an extreme slim disk (ESD) with a hot corona around a massive black hole with dimensionless accretion rate $\dot{m}$ in the range $2.5 \ll \dot{m} \leq 100$. We assume that a fraction $f$ of the gravitational energy is released in the hot corona and the rest is released in the ESD. The energy equation of the ESD is dominated by advection and the spectrum shows a broad "hump" caused by saturated Comptonization with monochromatic luminosity given by $v L_{v} \propto v^{0}$. This relationship enables us to estimate the black hole mass from the ESD luminosity. The spectrum of the hot corona is sensitive to the parameter $f \dot{M}$ and the viscosity and shows a Comptonized power-law with a high-energy cutoff. The model is used to explain the spectral properties of narrow line Seyfert 1 galaxies (NLS1s). In particular, it can explain the spectrum of extreme NLS1s like NLS1 RE J1034+396. Our spectral estimate of the black hole mass in this source is in good agreement with the mass obtained by applying the reververation mapping correlation. We also examine the Eddington ratios in a large NLS1 sample and find that most objects show super-Eddington accretion rates. We argue that soft X-ray humps in NLS1s are natural consequences of super-Eddington accretion in such objects.
\end{abstract}

Key words. accretion, accretion disks - galaxies: active - galaxies: Seyfert - X-rays: galaxies

\section{Introduction}

Narrow line Seyfert 1 galaxies (NLS1) receive much attention because of their unusual properties: the $\mathrm{H} \beta$ line is narrow $\left(F W H M \mathrm{H} \beta<2000 \mathrm{~km} \mathrm{~s}^{-1}\right.$ ) and relatively weak (less than a third of the intensity of the [OIII] $\lambda 5007$ line), and the optical FeII lines are very strong. These characteristics locate NLS1s near the extreme value of the Boroson \& Green (1992) Eigenvector 1. Soft X-ray excess and the tendency of the spectrum to flatten at low energy have been found in several NLS1s, e.g. PKS 0558-504 (O'Brien et al. 2001), Mrk 766 (Boller et al. 2001), PG 1244+026 (Fiore et al. 1998), PG 1404+226, PG 1440+356, PG 1211+143 (George et al. 2000), IRAS 13224-3809 (Vaughan et al. 1999), 1H 0707-495 (Boller et al. 2002; Dewangan et al. 2002), RX J1702.5+3247 (Gliozzi et al. 2001) and Ton S180 (Turner et al. 2001a; Turner et al. 2002). BeppoSAX and ASCA observations of Akn 564 and RE J1034+396 clearly show these extreme properties including a soft X-ray hump with a very flat spectrum $\left(v F_{v}=\right.$ const., Comastri et al. 2001; Puchnarewicz et al. 2001; Turner et al. 2001b). Other unusual X-ray properties are discussed in Brandt et al. (1998), Comastri et al. (2001), Pounds et al. (1995)

Send offprint requests to: J.-M. Wang, e-mail: wang@astro.uni-tuebingen.de and Collinge et al. (2001). Earlier suggestions attributing the soft excess to blends of emission lines are not supported by recent ASCA, XMM and Chandra observations (e.g. Turner et al. 1999; Puchnarewicz et al. 2001; Turner et al. 2001a,b; Collinge et al. 2001).

According to some theories, NLS1s contain relatively small (considering their luminosity) black holes (BHs) with very high accretion rates (Laor et al. 1994, 1997; Boller et al. 1996) exceeding, in some cases, the critical Eddington rate (Mineshige et al. 2000; Collin et al. 2002). This hypothesis is supported by comparing NLS1 spectra with the spectrum of Galactic black hole candidates (Pounds et al. 1995), and by very few direct measurements of the BH mass in a handful of NLS1s (e.g. Peterson et al. 2000). The observed properties of NLS1s, in particular the soft X-ray excess and the small BH mass, suggest that they may produce much of their emission due to processes in high accretion-rate disks with hot coronae. It is therefore important to calculate models of such systems.

Thin accretion disks are thought to produce the optical-UV continuum radiation in Broad Line Seyfert 1 galaxies (BLS1s; see Shields 1978; Malkan \& Sargent 1982; Malkan 1983; Laor $\&$ Netzer 1989). Indeed, thin disk spectra, based on the standard disk model (Shakura \& Sunyeav 1973) with Eddington ratio $L / L_{\mathrm{Edd}}<0.3$, give reasonable fits to the spectrum of many 
Table 1. Model list of emergent spectrum from accretion disk with high accretion rate.

\begin{tabular}{lllccccc}
\hline \hline reference & disk struc. & radiation & Comp. & $z$-structure & GR & corona & note \\
\hline CT95 & global & BB, f-f & Yes & No & No & No & shocks(?) \\
SAM96 & global & MBB & No & No & No & No & \\
WSLZ99 & global & f-f, BB & No & Yes & No & No & \\
MKTH00 & global & BB & No & No & No & No & \\
WN & self-similar & f-f, BB & Yes & No & No & Yes & \\
\hline
\end{tabular}

CT95: Chakrabarti \& Titarchuk (1995); SAM96: Szuszkiewicz et al. (1996); WSLZ99: Wang et al. (1999); MKTH00: Mineshige et al. (2000); $\mathrm{WN}$ - the present work.

f-f: free-free emission, BB: black body radiation, MBB: modified black body radiation by electron scattering, Comp.: comptonization; GR: general relativity.

BLS1s (Czerny \& Elvis 1987; Wandel \& Petrosian 1988; Sun \& Malkan 1989; Laor \& Netzer 1989; Laor 1990; Ross et al. 1993; Störzer et al. 1994; Shimura \& Takahara 1994; Dörrer et al. 1996; Sincell \& Krolik 1997; Hubeny \& Hubeny 1997; Kawaguchi et al. 2001). However, there were very few attempts to calculate the spectrum of high accretion-rate disks which we call either "slim disks" (SDs, after Abramowicz et al. 1988) or "extreme slim disks" (ESDs), depending on their accretion rate. The few models that are available in the literature are shown in Table 1 where we list their more important ingredients. Some common properties regarding the emergent spectrum are the flattening at soft X-ray energies, the high energy cutoff which is almost independent of the accretion rate, and the fact that the total luminosity is saturated (Wang \& Zhou 1999; Wang et al. 1999; Fukue 2000; Mineshige et al. 2000).

Another ingredient in some disk models is a hot corona. If exists, such a corona must be complex, as is evident from the highly variable X-ray spectrum. The geometry and formation of the hypothetical corona are poorly understood (see a review by Collin 2001). There are several published models of standard, thin accretion disks with hot coronae (see Shapiro et al. 1976; Liang \& Price 1977; Svensson \& Zdzarski 1994; Janiuk \& Czerny 2000), but, so far, no theoretical model of a high accretion-rate disk with a hot corona.

In this paper we study ESDs with hot coronae in order to explain the X-ray spectrum of NLS1s. The paper is arranged as follows: Sect. 2 gives the basic equations and explains the method of calculations. Section 3 discusses the spectral properties of such systems and in Sect. 4 we examine the Eddington ratio in several observed systems and apply our model to the soft hump observed in RE J1034+396. Finally, in Sect. 5 we discuss implications to future models and observations of NLS1s.

\section{The structure of ESDs with hot coronae}

\subsection{Extreme slim disks}

The standard accretion disk model (Shakura \& Sunyeav 1973) breaks down when the accretion rate approaches the Eddington rate. At this limit, the disk becomes "slim" and radial advection cannot be neglected any more. A large range of accretion rates $(\dot{M})$ and relative rates $(\dot{m})$ of such systems has been considered (Abramowicz et al. 1988; Szuszkiewicz et al. 1996;
Wang et al. 1999; Mineshige et al. 2000; Watarai \& Mineshige 2001; Ohsuga et al. 2002). Here

$\dot{m}=\frac{\dot{M}}{\dot{M}_{\mathrm{cr}}} ; \quad \dot{M}_{\mathrm{cr}}=\frac{64 \pi G M_{\mathrm{BH}} m_{\mathrm{p}}}{\sigma_{\mathrm{T}} c}$,

$\sigma_{\mathrm{T}}$ is the Thomson cross section, $m_{\mathrm{p}}$ the mass of the proton, $c$ the speed of light, $M_{\mathrm{BH}}$ the BH mass and we have assumed an accretion conversion efficiency of $\eta_{0}=1 / 16$. ESDs are taken here to be those cases with $2.5 \ll \dot{m} \leq 100$ (see discussions below). In such disks, the emitted photons are trapped in the gas due to the large Thomson depth which causes the diffusion time to exceed the radial motion time (Begelman \& Meier 1982; Wang \& Zhou 1999; Spruit 2000; see Ohsuga et al. 2002 for a more sophisticated treatments of photon trapping). In such cases, the surface cooling is less efficient with important consequences to the emergent spectrum. Below we consider ESDs where advection cooling dominates over other surface cooling due to such trapping.

In this work we consider also a hot corona that modifies the emergent disk spectrum by Compton scattering, and contributes to the high energy radiation. Previously published diskcorona models were based on the following assumptions: 1) a fraction $f$ of the gravitational energy is released in a hot corona and $(1-f)$ is deposited in the cold disk (Haardt \& Maraschi 1991). 2) the vertical equilibrium holds in both the cold disk and the hot corona and is described by the vertically averaged equations (Svensson \& Zdzarski 1994; Janiuk \& Czerny 2000). 3 ) the hot corona is cooled by Comptonization of soft photons emitted by the cold disk including the reflected photons. With these assumptions, the structure and the spectrum of slim disks with hot coronae can be obtained with the parameter $f$. There is no way to accurately find the value of $f$ which depends on the connection between the disk and the corona. We follow a simple prescription and treat $f$ as a free parameter of the model. The accretion rate in the ESD is thus $(1-f) \dot{M}$. There are other constraints on our model, such as the requirement of an optically thin corona (see below).

The basic ESD equations are similar to the SD equations for a steady disk in a Newtonian potential of azimuthal symmetry and Keplerian angular velocity $\Omega_{\mathrm{k}}$. We take the kinematic coefficient of the shear viscosity to be $v=\alpha a_{\mathrm{s}} H_{\mathrm{d}}$, where $a_{\mathrm{s}}$ is the sound speed, $\alpha$ the viscosity parameter, and $H_{\mathrm{d}}$ the half thickness of the disk. The basic equations are those proposed 
by Muchotrzeb \& Paczynski (1982), Matsumoto et al. (1984) and Abramowicz et al. (1988), except that we neglect the coefficients $B_{i}$ (which are of order unity) and the accretion rate is replaced by $(1-f) \dot{M}$. This gives

$(1-f) \dot{M}=4 \pi R H_{\mathrm{d}} \rho_{\mathrm{d}} v_{\mathrm{r}}^{\mathrm{d}}$,

$\frac{P_{\mathrm{d}}}{\rho_{\mathrm{d}}}=H_{\mathrm{d}}^{2} \Omega_{\mathrm{k}}^{2}$,

$(1-f) \dot{M}\left(l-l_{\text {in }}\right)=4 \pi R^{2} H_{\mathrm{d}} \alpha P_{\mathrm{d}}$,

$\frac{1}{\rho_{\mathrm{d}}} \frac{\mathrm{d} P_{\mathrm{d}}}{\mathrm{d} R}-\left(\Omega^{2}-\Omega_{\mathrm{k}}^{2}\right) R+v_{\mathrm{r}}^{\mathrm{d}} \frac{\mathrm{d} v_{\mathrm{r}}^{\mathrm{d}}}{\mathrm{d} R}+\frac{P_{\mathrm{d}}}{\rho_{\mathrm{d}}} \frac{\mathrm{d} \ln \Omega_{\mathrm{k}}}{\mathrm{d} R}=0$,

where $P_{\mathrm{d}}$ is the total pressure, $\rho_{\mathrm{d}}$ the mass density, $v_{\mathrm{r}}^{\mathrm{d}}$ the radial velocity of the flow, $\Omega$ the angular velocity, $l$ the specific angular momentum, and $l_{\text {in }}$ the eigen value of angular momentum at the inner boundary. The last term in Eq. (5) is the correction for the decrease of the radial component of the gravitational force away from the equator (Matsumoto et al. 1984).

While the above equations are suitable to describe the slim disk-corona coupling, such calculations are beyond the scope of the present paper. We only consider Thomson thin coronae in the limit $f \ll 1$. Despite the small $f$, the hot corona can be a strong emitter since $f \dot{M}$ is not necessarily small.

The energy conservation equations should be added to the above equations. Advection cooling, which can dominate over the diffusion cooling, is given by

$Q_{\mathrm{adv}}=-T_{\mathrm{d}} \frac{(1-f) \dot{M}}{4 \pi R} \frac{\mathrm{d} S}{\mathrm{~d} R}=\frac{(1-f) \dot{M}}{4 \pi R^{2}}\left(\frac{P_{\mathrm{d}}}{\rho_{\mathrm{d}}}\right) \xi$,

(Abramowicz et al. 1988) where $S$ is the entropy,

$\xi=(4-3 \beta) \gamma_{\rho}-(12-10.5 \beta) \gamma_{\mathrm{T}}$,

$\gamma_{\rho}=\mathrm{d} \ln \rho_{\mathrm{d}} / \mathrm{d} \ln R, \gamma_{\mathrm{T}}=\mathrm{d} \ln T_{\mathrm{d}} / \mathrm{d} \ln R$ and $\beta$ is the ratio of gas to total pressure. In this paper we take $\beta=0$ since radiation pressure dominates over gas pressure. Thus, $\xi=4 \gamma_{\rho}-12 \gamma_{\mathrm{T}}$. For intermediate values of $f$, the energy equation is $Q_{\mathrm{adv}}+Q_{\mathrm{cool}}^{\mathrm{d}}=$ $Q_{\mathrm{vis}}^{\mathrm{d}}$, where $Q_{\mathrm{vis}}^{\mathrm{d}}$ is the energy generation via viscosity in the SD and $Q_{\text {cool }}^{\mathrm{d}}$ is the cooling from the surface of the SD. For ESDs, the condition $f \ll 1$ guarantees that advection is the dominant cooling process $\left(Q_{\mathrm{adv}} \gg Q_{\mathrm{cool}}^{\mathrm{d}}\right)$ and practically balances $Q_{\text {vis }}^{\mathrm{d}}$, i.e.,

$Q_{\mathrm{adv}}=Q_{\mathrm{vis}}^{\mathrm{d}}=(1-f) Q_{\mathrm{vis}}$

where $Q_{\text {vis }}$ is the energy generated by viscosity per unit area

$Q_{\text {vis }}=\frac{\dot{M}\left(l-l_{\text {in }}\right)}{4 \pi R}\left(-\frac{\mathrm{d} \Omega}{\mathrm{d} R}\right)=\frac{n \mathcal{J} \dot{M} \Omega^{2}}{4 \pi}$,

where $n=-\mathrm{d} \ln \Omega / \mathrm{d} \ln R$ and $\mathcal{J}=1-l_{\text {in }} / l$. Strictly speaking, the radial motion and energy conservation equations can be solved given the appropriate boundary conditions (Abramowicz et al. 1988). Denoting $\gamma_{\mathrm{p}}=\mathrm{d} \ln P_{\mathrm{d}} / \mathrm{d} \ln R$, and $\gamma_{v}=\mathrm{d} \ln v_{\mathrm{r}}^{\mathrm{d}} / \mathrm{d} \ln R$, the radial motion Eq. (5) is reduced to an algebraic equation

$\left(\gamma_{\mathrm{p}}-\frac{3}{2}\right) \frac{P_{\mathrm{d}}}{\rho_{\mathrm{d}}}-\left(\Omega^{2}-\Omega_{k}^{2}\right) R^{2}+\gamma_{v}\left(v_{\mathrm{r}}^{\mathrm{d}}\right)^{2}=0$.
Neglecting the boundary condition, i.e., setting $\mathcal{J}=1$ (or $l_{\text {in }} \approx 0$ ), and using Eqs. (2), (3), (4), (8) and (10), we get, after some algebraic manipulations, the following set of self-similar solutions:

$P_{\mathrm{d}}=\frac{(1-f) \dot{M} \Omega_{k}}{4 \pi \alpha R}, \rho_{\mathrm{d}}=\frac{\gamma_{0}^{2}}{4 \pi \alpha} \frac{(1-f) \dot{M}}{\Omega_{k} R^{3}}$,

$v_{\mathrm{r}}^{\mathrm{d}}=\alpha \gamma_{0}^{-1} R \Omega_{k}, \quad H_{\mathrm{d}}=\gamma_{0}^{-1} R$,

$\Omega=\frac{\Omega_{k}}{\gamma_{0}}, a_{\mathrm{s}}=\frac{R \Omega_{k}}{\gamma_{0}}$,

where $\gamma_{0}=\left(5+\alpha^{2} / 2\right)^{1 / 2}$ is a weak function of the viscosity $\alpha$ which is roughly $\sqrt{5}$ for $\alpha \ll 1$. It is interesting to note that $v_{\mathrm{r}}^{\mathrm{d}}$, $H_{\mathrm{d}}, \Omega$, and $a_{\mathrm{s}}$ are independent of $f$. When $f$ goes to zero, the solution reduces to the one given in Wang \& Zhou (1999).

The usual treatment of the inner edge of the accretion disk assumes a certain last stable orbit, $R_{\mathrm{ms}}$, depending on the $\mathrm{BH}$ properties (e.g. $R_{\mathrm{ms}}=3 R_{\mathrm{S}}$ for a Schwarzschild black hole with $\left.R_{\mathrm{s}}=2 G M_{\mathrm{BH}} / c^{2}\right)$. This is also taken to be the innermost emitting radius $R_{\text {in }}$. Most of the calculations presented here assume a Schwarzschild BH with the corresponding inner radius. However, Mineshige et al. (2000) and Watarai \& Mineshige (2001) have shown that a substantial amount of radiation can be produced from inside this value of $R_{\mathrm{ms}}$ if the accretion rate is larger than $5 \dot{M}_{\mathrm{c}}$. These authors take $R_{\text {in }}$ to be a free parameter of the model. Moreover, it has been argued (Krolik \& Hawley 2002) that in real disks, $R_{\text {in }}<R_{\mathrm{ms}}$, i.e. a substantial fraction of the observed radiation is emitted inside the last stable orbit. Our calculations cannot include the Krolik and Hawley effects and we have adopted the general approach of a free $R_{\text {in }}$ as in Mineshige et al. (2000). We note that the smaller $R_{\text {in }}$ is, the better is the self-similar approximation since, as explain, for this condition to hold we require $\mathcal{J} \simeq 1$.

While surface cooling in ESDs is very small compared with the advected energy, the diffuse photons play an important role in cooling the corona. We can calculate $Q_{\text {cool }}^{\mathrm{d}}$ via the diffusive approximation. The temperature at the central plane of an ESD is given by $T_{\mathrm{d}}=\left(3 P_{\mathrm{d}} / a\right)^{1 / 4}=6.69 \times 10^{7}[(1-$ f) $\dot{m} / m]^{1 / 4} \alpha^{-1 / 4} r^{-5 / 8} \mathrm{~K}$, where $a$ is the radiation density constant and the effective temperature is $T_{\text {eff }} \approx T_{\mathrm{d}} / \tau_{\mathrm{es}}^{1 / 4}$ (Laor \& Netzer 1989). For Thomson scattering, $\tau_{\mathrm{es}}=\sigma_{\mathrm{T}} \rho_{\mathrm{d}} H_{\mathrm{d}}$, leading to

$T_{\text {eff }}=3.64 \times 10^{7} \gamma_{0}^{-1 / 4} m_{\mathrm{BH}}^{-1 / 4} r^{-1 / 2} \mathrm{~K}$,

where $m_{\mathrm{BH}}=M_{\mathrm{BH}} / M_{\odot}$, and $r=R / R_{\mathrm{S}}$. We thus get an effective temperature distribution of the form $T_{\text {eff }} \propto r^{-1 / 2}$, as in Wang \& Zhou (1999) and Fukue (2000). This radial dependence is caused mostly by photon trapping. The surface energy flux, $Q_{\text {cool }}^{\mathrm{d}}$, is

$Q_{\text {cool }}^{\mathrm{d}}=\sigma T_{\text {eff }}^{4}=1.0 \times 10^{26} \gamma_{0}^{-1} m_{\mathrm{BH}}^{-1} r^{-2} \mathrm{erg} \mathrm{s}^{-1} \mathrm{~cm}^{-2}$.

We will show later that the black body approximation used here to calculate the emergent flux is justified because of the saturated Comptonization in ESDs.

It is important to note that $T_{\text {eff }}$ and $Q_{\text {cool }}^{\mathrm{d}}$ are independent of the accretion rate and the factor $f$ but depend on the mass of 
the black hole and very weakly on the viscosity $\alpha$ through $\gamma_{0}$. Thus we can estimate the BH mass directly from the spectrum.

In ESDs, the time scale of photon diffusion in the $z$ direction is $\tau_{\text {diff }} \sim H_{\mathrm{d}} \kappa_{\mathrm{es}} \Sigma / c$ whereas the radial transportation time scale of the accreting gas is $\tau_{\mathrm{R}} \sim \pi R^{2} \Sigma / \dot{M}$ (Mineshige et al. 2000). The condition $\tau_{\mathrm{R}}=\tau_{\text {diff }}$ can be used to obtain the dimensionless photon trapping radius, inside which the photons will be trapped in the gas,

$r_{\text {trap }}=7.2 \times 10^{2}(1-f)\left(\frac{\dot{m}}{50}\right)$.

The total ESD luminosity integrated over the photon trapping region is obtained from the integration of Eq. (15)

$L_{\mathrm{rad}} \approx 8.0 \times 10^{37} m_{\mathrm{BH}} \ln \left[\frac{(1-f) \dot{m}}{50}\right] \mathrm{ergs} \mathrm{s}^{-1}$.

We see that the total radiated luminosity $L_{\text {rad }}$ is only weakly dependent on the accretion rate $\dot{m}$. Comparing with the thin disk critical radius $r_{\mathrm{h}} \approx 14$ (Laor \& Netzer 1989), inside which half the total luminosity is dissipated, we see that for high accretion rates, most of the gravitational energy is released within the trapping radius and much of it is advected into the black hole. Because of that, the calculations of the ESD spectrum neglect the region beyond $r_{\text {trap }}$ that contributes very little to the emergent spectrum.

A necessary condition for our self-similar solution to hold is $Q_{\mathrm{adv}} \gg Q_{\mathrm{cool}}^{\mathrm{d}}$. This is equivalent to $\dot{m} \gg 0.18 r$. If we demand that advection dominates surface cooling at $r=r_{\mathrm{h}}$, namely at least half the dissipated energy will be advected, we get $\dot{m} \gg 2.5$ which, using eqn. 16 gives $r_{\text {trap }} \approx 36$. As explained, such a high accretion rate leads to a significant release of gravitational energy inside the last stable orbit. Thus, in our model we allow $R_{\text {in }}<3 R_{\mathrm{s}}$.

\subsection{The hot corona}

We assume that the gas in the optically thin corona rotates with Keplerian velocity, and that gas pressure dominates over radiation pressure since the corona is optically thin. Advection is mainly due to protons that are heated close to the virial temperature while the temperature of the electrons is much lower due to the inefficient energy exchanges between electrons and protons and due to Comptonization cooling. The assumption that vertical equilibrium holds means that the height of the corona $\left(H_{\mathrm{c}}\right)$ is given by $H_{\mathrm{c}}=c_{\mathrm{s}} / \Omega_{k}$, where $c_{\mathrm{s}}=\left(P_{\mathrm{c}} / \rho_{\mathrm{c}}\right)^{1 / 2} \propto$ $T_{\mathrm{p}}^{1 / 2}$ is the sound speed. The cooling in the corona is determined by Comptonization, and the seed photons are contributed from two sources: 1) direct emission from the ESD and 2) ESD reprocessing of photons scattered down by the hot corona. Considering the fraction $\eta$ of the coronal flux directed towards the ESD, and the energy averaged albedo $a$, Comptonization cooling can be written as

$Q_{\mathrm{c}}=A\left[Q_{\mathrm{cool}}^{\mathrm{d}}+\eta(1-a) Q_{\mathrm{c}}\right]$,

where $A=\exp (y)-1$ is the Compton amplification factor, $y=$ $\tau_{\mathrm{es}} \Xi_{\mathrm{e}}\left(1+\Xi_{\mathrm{e}}\right)$, and $\Xi_{\mathrm{e}}=4 k T_{\mathrm{e}} / m_{\mathrm{e}} c^{2}$. Following Życki et al. (1995), we assume

$Q_{\mathrm{c}}+Q_{\mathrm{adv}}^{\mathrm{c}}=\frac{3}{2} \Omega_{k} \alpha_{\mathrm{c}} P_{\mathrm{c}} H_{\mathrm{c}}$ where $\alpha_{\mathrm{c}}$ is the viscosity parameter in the corona, which may be different from the viscosity in the ESD. $Q_{\mathrm{adv}}^{\mathrm{c}}$ is the advected energy due to the protons in the corona,

$Q_{\mathrm{adv}}^{\mathrm{c}}=\frac{f \dot{M} c_{\mathrm{s}}^{2}}{4 \pi R^{2}} \delta(R)$

where $\delta(R)=\mathrm{d} \ln P_{\mathrm{c}} / \mathrm{d} \ln R-2.5 \mathrm{~d} \ln T_{\mathrm{p}} / \mathrm{d} \ln R$ is a parameter controlling the corona advection (Janiuk \& Czerny 2000) that should be obtained self-consistently since it is a function of radius (see next section). The energy equation in the hot corona can be written as

$Q_{\mathrm{c}}+Q_{\mathrm{adv}}^{\mathrm{c}}=f Q_{\mathrm{vis}}$.

As explained, in such an optically thin medium, the electron and proton temperatures can be quite different due to inefficient energy exchange. Thus, the hot corona is a two-temperature plasma. The net flux from protons to electrons is given by

$Q_{\mathrm{c}}=1.5 \mathrm{~km}_{\mathrm{p}}^{-1}\left(T_{\mathrm{p}}-T_{\mathrm{e}}\right)\left(1+\Xi_{\mathrm{e}}^{1 / 2}\right) v_{\mathrm{ei}} \rho_{\mathrm{c}} H_{\mathrm{c}}$,

where $v_{\mathrm{ei}}=2.44 \times 10^{21} \rho_{\mathrm{c}} T_{\mathrm{e}}^{-1.5} \ln \Lambda$ with $\ln \Lambda \approx 20$ (Shapiro et al. 1976). We take $\eta=0.5$ and $a=0.2$ throughout this paper. The free-free emission is $j_{v} \approx 6.8 \times 10^{-38} n_{\mathrm{e}}^{2} T_{\mathrm{e}}^{-0.5}$ (we neglect the Gaunt factor). The ratio of $\mathrm{f}-\mathrm{f}$ emission to Comptonization obtained from Eq. (22) is $5.6 v_{19}\left(T_{\mathrm{e}} / T_{\mathrm{p}}\right) \ll 1$ if $T_{\mathrm{e}} \ll T_{\mathrm{p}}$, where $v_{19}=v(\mathrm{~Hz}) / 10^{19}$. Thus free-free emission can be neglected in the two-temperature plasma. The structure of the hot corona is determined by viscosity heating, and advection and Comptonization cooling.

An important constraint on the value of $f$ is obtained from limits on the Thomson depth of the corona. The number density near the last stable orbit $\left(R_{\mathrm{ms}}\right)$ is $n_{\mathrm{c}}=f \dot{M} / 4 \pi R_{\mathrm{ms}}^{2} c m_{\mathrm{p}}$, where we take the radial velocity to be the speed of light and the height of the corona to be $R_{\mathrm{ms}}$. The Thomson depth is thus $\tau_{\mathrm{c}}=n_{\mathrm{c}} \sigma_{\mathrm{T}} R_{\mathrm{ms}}=f \dot{m} / 6 \eta_{0}$. This means that $f<6 \eta_{0} \dot{m}^{-1}$ or else the corona is Thomson thick (Blandford 1990). This constraint does not depends on the disk model. To summarize, the present model is valid when $\dot{m} \gg 2.5$ and $f<0.0375(\dot{m} / 10)^{-1}$.

The structure of ESDs with hot coronae is controlled by six parameters: $\alpha, \dot{m}, m_{\mathrm{BH}}, r_{\mathrm{in}}, \alpha_{\mathrm{c}}$ and $f$. The first four describe the ESD and the other two the hot corona. It is important to note that the density and the height of the corona depend on the black hole mass, but the corona structure, i.e. $T_{\mathrm{e}}, T_{\mathrm{p}}$, and $\tau_{\mathrm{es}}$, is independent of the black hole mass when using the dimensionless radius $r$. This implies that the corona structure will be similar in stellar and super-massive black hole accreting systems. The corona structure is also only weakly dependent on the accretion rate. These may be the reasons why the hard $\mathrm{X}$-ray spectrum of Galactic black hole candidates at high state is quite similar to that of narrow line Seyfert 1 galaxies containing much larger black holes.

We used an iteration method similar to Chen (1995) and Janiuk \& Czerny (2000) to calculate advection in the hot corona. In this procedure, an initial value of advection factor $\delta(R)$ at radius $R$ is assumed. We then obtain the structure of the corona at $R-\Delta R / 2$ and $R+\Delta R / 2$ and a new $\delta(R)$ which is used for the next iteration until the value of $\delta$ converges. In practice, the iteration converges very fast. 

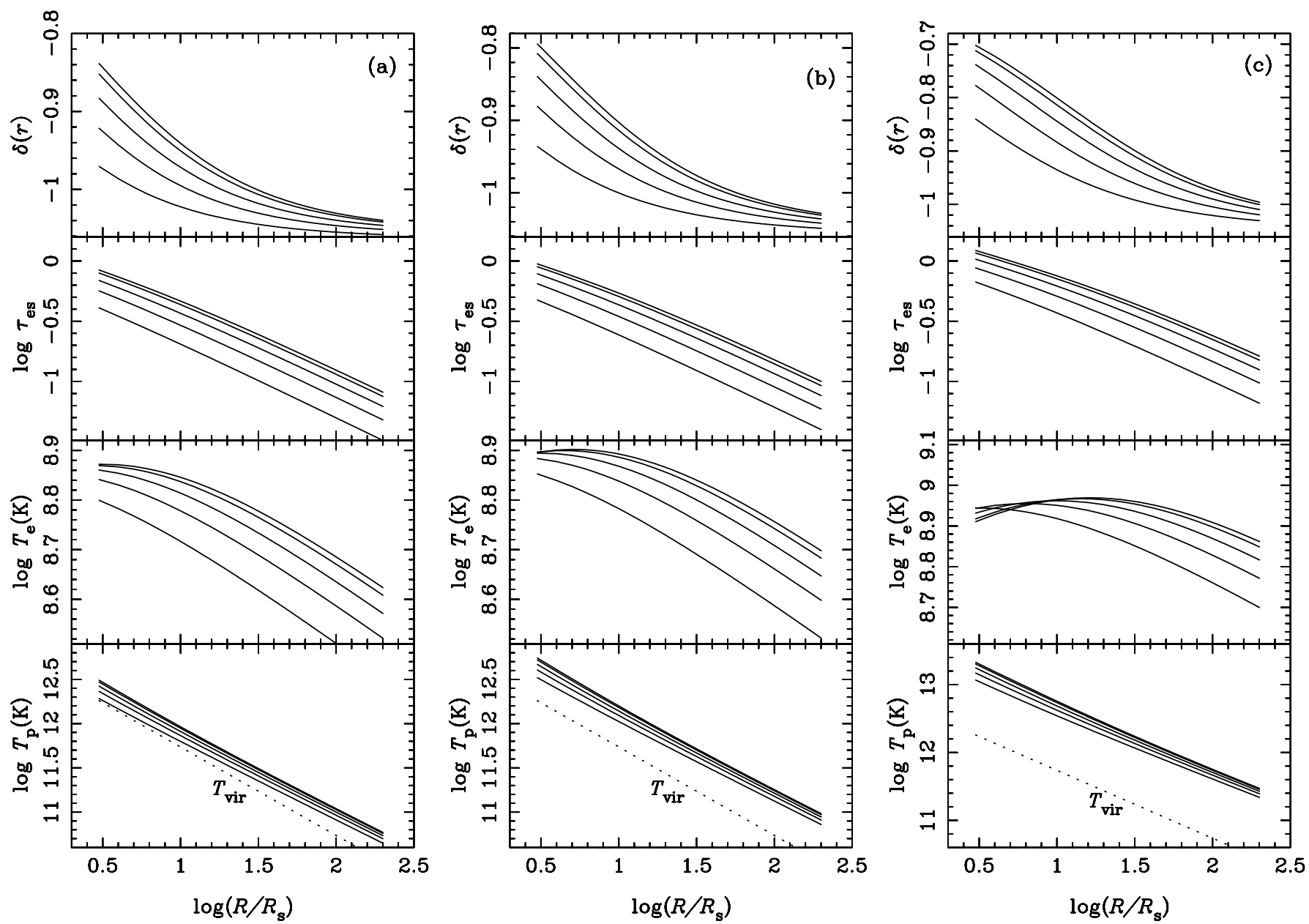

Fig. 1. The structure of hot corona above ESD. The accretion rates, from bottom to top, are $\dot{m}=30,50,70,90,100 \dot{M}_{\mathrm{cr}}$ and $f=10^{-3}$. In a) $\alpha=\alpha_{\mathrm{c}}=0.1$, in b) $\alpha_{\mathrm{c}}=0.05$ and in c) $\alpha_{\mathrm{c}}=0.01$. The advective parameter $\delta(r)$ is obtained from self-consistent iterations (see text). The hot corona becomes optically thick when $\dot{m}>100$ (for $f=10^{-3}$ ). Under such conditions, emission from the disk is blocked and no hump is observed. Using the dimensionless radius $r\left(=R / R_{\mathrm{S}}\right)$, the structure of the corona is independent of the mass of the black hole.

Figures 1a-1c show the structure of a hot corona above an ESD for various accretion rates $\dot{M}_{\mathrm{c}}=f \dot{M}$ and viscosity $\alpha$. From Fig. 1a, we see that the Thomson depth $\left(\tau_{\text {es }}\right)$, electron temperature $\left(T_{\mathrm{e}}\right)$ and proton temperature $\left(T_{\mathrm{p}}\right)$ increase with $\dot{M}_{\mathrm{c}}$. If we let the factor $f$ increase, the corona will gradually become optically thick and blocks the emission from the ESD. This gives a constraint on $f(f \ll 1)$. The temperature of the electrons in the corona is a weak function of radius. The optical depth $\tau_{\text {es }}$ is a power law in radius, and is sensitive to the accretion rate $f \dot{M}$.

We find that the proton's temperature is super-virial, $T_{\text {vir }}=$ $5.45 \times 10^{12} r^{-1} \mathrm{~K}$. This temperature is caused by advection, in which the energy dissipated by viscosity is stored as entropy rather than being radiated. Such a process is enhanced by the inefficient energy exchange between electrons and protons. Advection affects the corona structure, and is found to be a source of heating rather than cooling. We also see that $\delta(r)$ tends to a constant at larger radii.

We tested the effect of changing the viscosity on the corona structure (Figs. 1b, 1c). We find that the scattering depth $\tau_{\mathrm{es}}$ increases slightly with decreasing $\alpha_{\mathrm{c}}$ due to the decrease of radial velocity with $\alpha_{\mathrm{c}}$, resulting in the increases of scattering depth.
For a hot corona with lower viscosity and higher accretion rate, we find that the location of maximum electron temperature is shifting to very large radii (e.g. $\log r \sim 1.5$ ). These effects have no significant observational consequences.

\section{The spectrum of ESDs with hot coronae}

We have calculated spectra of ESDs with hot coronae over a large range of $\mathrm{BH}$ mass, accretion rate and relative accretion parameter $f$. Like previous works, we find Comptonization to be very significant in such systems. The importance of the process can be estimated by considering the parameter

$Y=\Xi_{\mathrm{e}}\left(\frac{\kappa_{\mathrm{es}}}{\kappa_{\mathrm{ff}}}\right)$,

where the Thomson opacity is $\kappa_{\mathrm{es}}=0.34$, the free-free opacity is $\kappa_{\mathrm{ff}}=1.5 \times 10^{25} \rho T^{-7 / 2} x^{-3}\left(1-\mathrm{e}^{-x}\right)$, and $x=h v / k T$. To evaluate $Y$, we use the self-similar solution (Eqs. (11)-(13)). The results are shown in Fig. 2 for $\dot{m}=30$. We find $Y \gg 1$ for high energy photons $(x>0.2)$ in a large region inside the trapping radius, suggesting saturated Comptonization. For higher accretion rate, $Y$ is even larger, and Comptonization is 


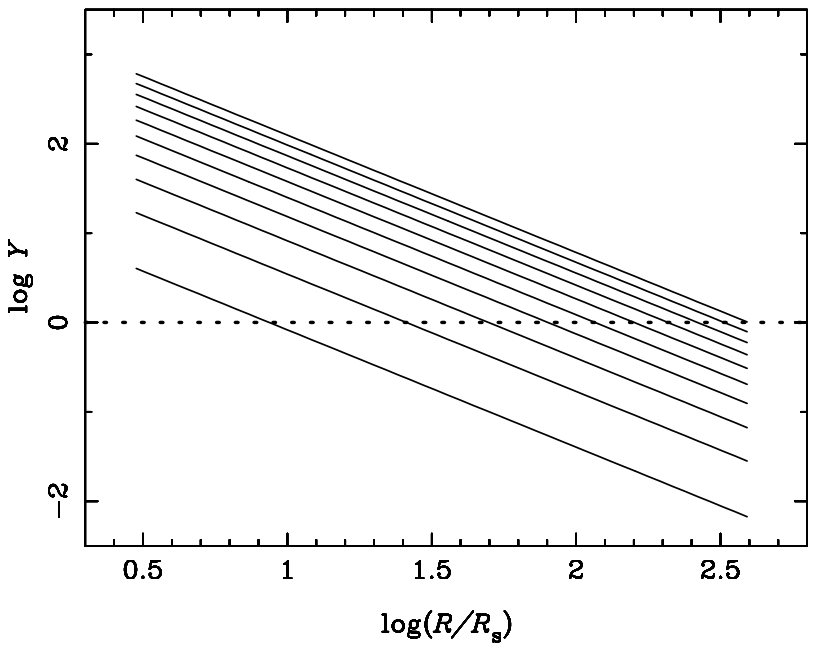

Fig. 2. The Comptonization parameter $Y$ for $\dot{m}=30.0$ and $\alpha=0.1$. The solid lines, from bottom to top are: $x=h v / k T=0.1,0.2,0.3,0.4$, $0.5,0.6,0.7,0.8,0.9,1.0$. Comptonization is highly saturated above the dotted line that indicates $Y=1.0$.

stronger. Wang et al. (1999) and Mineshige et al. (2000) considered the effects of advection but neglected Comptonization in their calculations. The present paper includes all these processes. Figure 2 shows the Comptonization parameter $Y$ as a function of the normalized radius.

With high accretion rate, ESDs have unique effective temperature distribution of the form $T_{\text {eff }} \propto r^{-1 / 2}$ and relatively high temperature. The emergent spectrum differs significantly from the spectrum of thin accretion disks with or without coronae. We use the method described by Czerny \& Elvis (1987) (see also Wandel \& Petrosian 1988) for the spectral calculations. The disk structure is self-similar (Eqs. (11)-(13)) and the surface temperature $T_{\mathrm{S}}$ is taken to be $T_{\text {eff }}$ from Eq. (14). The ESD spectrum is given by integrating over the photon trapping region,

$L_{v}=\pi \int_{R_{\mathrm{in}}}^{R_{\mathrm{tr}}} B_{v}\left(T_{\mathrm{s}}\right) f_{v} R \mathrm{~d} R$

where the factor $f_{v}$ describes the departure from black body radiation, including Comptonization,

$f_{v}=f_{\mathrm{ff}}(v)\left[1-f_{\mathrm{th}}(v)\right]+C$.

Here the factors $f_{\mathrm{ff}}$ and $f_{\text {th }}$ are given by

$f_{\mathrm{ff}}=\frac{2\left[1-\mathrm{e}^{-2 \tau_{v}^{*}}\right]}{1+\left(\kappa_{\mathrm{tot}} / \kappa_{\mathrm{abs}}\right)^{1 / 2}} ;$

and

$f_{\mathrm{th}}=\exp \left[-\frac{\ln \left(k T_{s} / h v\right)}{\tau_{\mathrm{es}}^{2} \ln \left(1+\Xi_{\mathrm{e}}+\Xi_{\mathrm{e}}^{2}\right)}\right]$,

where $f_{\text {th }}$ is the fraction of thermalized photons, $\kappa_{\text {tot }}=\kappa_{\mathrm{es}}+\kappa_{\mathrm{ff}}$ is the total scattering and free-free opacity, $\tau_{v}^{*}=\left(\kappa_{\mathrm{tot}} \kappa_{\mathrm{ff}}\right)^{1 / 2} \Sigma$ is the effective optical depth, and the Comptonization factor $C$ is given in Czerny \& Elvis (1987).

Regarding the hot corona, the emergent spectrum can be calculated at each radius from the semi-analytical formula in
Hua \& Titarchuk (1995). We assume that the photons are Comptonized locally, i.e. we neglect the Comptonization of seed photons from other radii. The integration is performed over the trapping radius.

Figure 3 shows spectra of ESDs with hot coronae for different accretion rates and black hole mass. The most prominent feature is a strong soft X-ray energy band. The luminosity of the hump is insensitive to the accretion rate because of photon trapping and increases only slightly as $\sim \ln \dot{m}$ (Eq. (17)). This results from the superposition of saturated Comptonized spectrum at different radii, satisfying the condition $Y \gg 1$ (Fig. 2). For such a large $Y$, the factor $f_{\text {th }}$ tends to 1 and $C$ tends to a constant (Eq. (25)). With the effective temperature distribution $T_{\text {eff }} \propto r^{-1 / 2}$, we get an emergent spectrum that satisfies the condition $v L_{\gamma} \propto v^{0}$ (Eq. (24)) and $L_{v}$ is proportional to $m_{\mathrm{BH}}$ (see below). This characteristic shape is in rough agreement with the no Comptonization case (Wang 1999; Wang et al. 1999; Mineshige et al. 2000), but the maximum frequency is at higher energy.

The accretion rate determines the trapping radius which determines also the shape of the red side of the soft hump. Comparing the cases of $\dot{m}=10$ and $\dot{m}=40$, we find that the width of the soft hump is greatly reduced at smaller $\dot{m}$. The behavior can be understood by inserting Eqs. (16) into (14) to get the minimum frequency of the hump

$v_{\min } \approx 2.82 \times 10^{16} \gamma_{0}^{-1 / 4} m_{\mathrm{BH}}^{-1 / 4}\left(\frac{\dot{m}}{50}\right)^{-1 / 2} \mathrm{~Hz}$

where we assume $f \approx 0$. The maximum frequency of the hump, which is determined by saturated Comptonization, is independent of $\dot{m}$ and is given by $v_{\max } \approx 2.7 k T_{\text {eff }}\left(r_{\text {in }}\right) / h$, namely

$v_{\max } \approx 1.19 \times 10^{18} \gamma_{0}^{-1 / 4} m_{\mathrm{BH}}^{-1 / 4}\left(\frac{r_{\mathrm{in}}}{3}\right)^{-1 / 2} \mathrm{~Hz}$.

The width of the soft hump, from $v_{\min }$ to $v_{\max }$, depends weakly on the accretion rate. This is very different from standard thin disk models where the maximum frequency and luminosity depends on both the $\mathrm{BH}$ mass and the accretion rate. The monochromatic luminosity in the flat part of the hump is not sensitive to the accretion rate but depends linearly on the mass of the black hole. This results in a good method to determine a black hole mass for super-Eddington accretion flows (see below).

The spectrum of the hot corona is determined by two parameters: $f \dot{m}$ and $\alpha_{\mathrm{c}}$. Several examples are shown in Fig. 3. The general shape is a power-law continuum with a cutoff. The spectrum becomes harder with increasing $f$. This dependence can be understood with the help of Figs. 1a-1c that show that the optical depth and temperature of the hot corona increase with $f$. The resulting spectrum can easily be understood by the simplest version of Comptonization. The temperature determines the cutoff energy whereas the photon index $p$ of the spectrum is determined by the scattering opacity and the temperature, $p=(\gamma+9 / 4)^{1 / 2}-1.5$, where $\gamma=4 \pi^{2} / 3\left(\tau_{\mathrm{es}}+2 / 3\right)^{2} \Xi_{\mathrm{e}}$ (Sunyaev \& Titarchuk 1980). When the accretion rate $\dot{M}_{\mathrm{c}}$ (or $f$ ) increases, both $\tau_{\mathrm{es}}$ and $\Xi_{\mathrm{e}}$ increase, resulting in the decrease of $\gamma$. Thus, the emergent spectrum becomes harder as the accretion rate increases. 


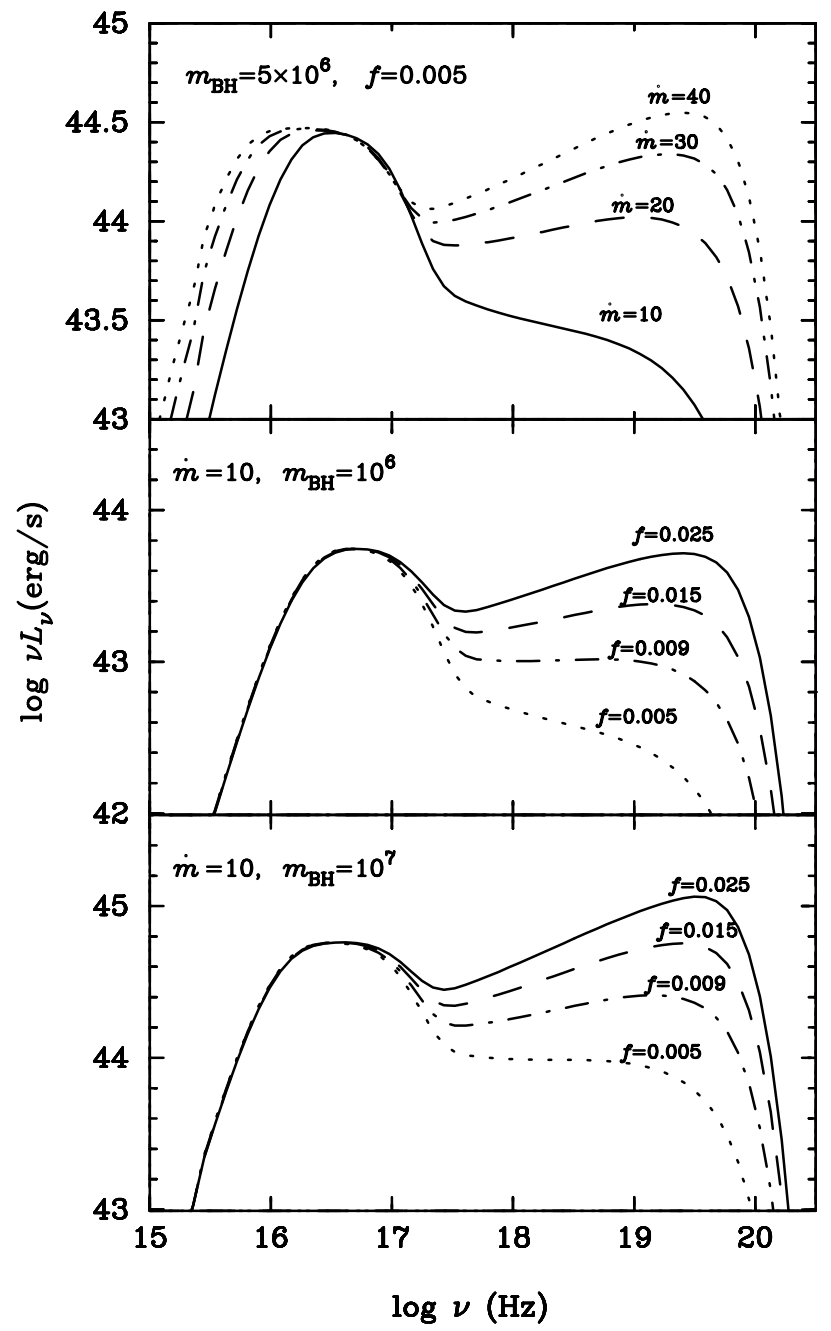

Fig. 3. The emergent spectrum from ESDs with hot coronae for different accretion rates, black hole masses and $f$. In all cases $\alpha=\alpha_{\mathrm{c}}=0.1$. The spectrum is composed of two components: the ESD contribution at low energy and the hot corona emission at high energy. The relative flux of the two depends on the value of $f$ and $\dot{m}$ which are marked on the diagram. Note the linear scaling of the hump luminosity with $m_{\mathrm{BH}}$.

The overall spectrum shows a "knee", a transiting from soft to hard X-rays. The flux at the knee frequency is contributed from the superposition of the Wien tail of the soft hump and the Comptonization of the soft hump. The knee's flux increases with $f$ and with decreasing viscosity and is correlated with the hard X-ray spectral index (Fig. 3). This is caused by the fact that the radiation from the hot corona increases with $f$. Finally, The cutoff energy of the hot corona spectrum depends on the maximum temperature of the electrons. This cutoff, which is below $1 \mathrm{MeV}$, may be observed by future missions such as INTEGRAL.

The radiation efficiency of an ESD (the fraction of the total gravitational energy lost to radiation) is low, $\eta_{\mathrm{ESD}}=$ $L_{\mathrm{rad}} / \eta_{0} \dot{M} c^{2} \approx 1.6 \times 10^{-2}(\dot{m} / 50)^{-1}$. The low efficiency implies that most of the photons are trapped and much of the gravitational energy dissipated by viscosity is advected into the $\mathrm{BH}$. We call such a flow with super-Eddington accretion rate "photon-trapping accretion flow" (PTAF).
The low conversion efficiency causes the hump luminosity to be weakly dependent on the accretion rate. Therefore, even with the uncertainties on $f$, the mass of the black hole can be estimated from the hump luminosity. Using Eq. (14) and integrating from the inner radius to the trapping radius we get

$M_{\mathrm{BH}}=2.8 \times 10^{6}\left(\frac{v L_{\nu}}{10^{44} \mathrm{erg} / \mathrm{s}}\right) M_{\odot}$,

where $v L_{v}$ is the luminosity anywhere in the flat part of the hump, and we have used the approximation $\int_{x_{\text {in }}}^{x_{\text {tra }}} x^{3} \mathrm{~d} x /\left(\mathrm{e}^{x}-\right.$ $1) \approx \int_{0}^{\infty} x^{3} \mathrm{~d} x /\left(\mathrm{e}^{x}-1\right)=\pi^{4} / 15, x_{\text {in }}=h v / k T\left(r_{\text {in }}\right)$, and $x_{\text {trap }}=h v / k T\left(r_{\text {trap }}\right)$. This method for determining the BH mass is unique since the the exact values of accretion rate and viscosity do not enter the mass determination. This method does not work for sub-critical accretion.

It is worth pointing out that in the context of standard accretion disks, under the assumption that the viscous stress is proportional to radiation pressure, the radiation pressuredominated region is thermally and viscous unstable because of the sensitive temperature dependence on viscosity. The optically thick ADAF has no such instability (under the $\alpha$ description) because the surface density $\Sigma$ is proportional to $\dot{m}$ (Eqs. (11)-(13)).

\section{Applications to NLS1s}

\subsection{The Eddington ratio in NLS1s}

There are several claims in the literature regarding the large $L / L_{\mathrm{Edd}}$ in NLS1s (e.g. Pounds et al. 1995; Boller et al. 1996; Laor et al. 1997; Collin et al. 2002). However, the available mass estimates are remarkably few and, in most cases, highly uncertain. In particular, detailed reverberation mapping for the purpose of measuring the $\mathrm{BH}$ mass, are available for only a handful of sources. Several big campaigns, such as the one described in Collier et al. (2001) and Shemmer et al. (2001), provided uncertain mass estimates because the observed continuum variability was very small. Currently there are seven objects with $F W H M(\mathrm{H} \beta)<1500 \mathrm{~km} \mathrm{~s}^{-1}$ and measured BH mass. Their observed properties are listed in Kaspi et al. (2000) and discussed further in Peterson et al. (2000). According to Peterson et al. (2000), the BH mass in those sources is considerably smaller than the masses measured in comparable luminosity BLS1s. This interpretation is still questionable because of the uncertain BLR size used in those mass estimates. Collin \& Huré (2001) and Collin et al. (2002) examined accretion rates in AGN using the Kaspi et al. (2000) relationship. They find that half the objects in their sample are accreting close to the Eddington rate or at a super-Eddington rate. The NLS1s in Collin's sample have the largest Eddington ratio.

To further test this idea, we have used the compilation of Véron-Cetty et al. (2001) that contains a heterogeneous sample of 54 NLS1s with $F W H M(\mathrm{H} \beta)<2000$ and $B$-band absolute magnitudes. The reverberation relation of Kaspi et al. (2000) allows a determination of $R_{\mathrm{BLR}}$ as a function of luminosity over a large range in optical continuum luminosity. Following Wang \& Lu (2001), we estimated BH masses in this sample using the $5100 \AA$ monochromatic luminosity which we 


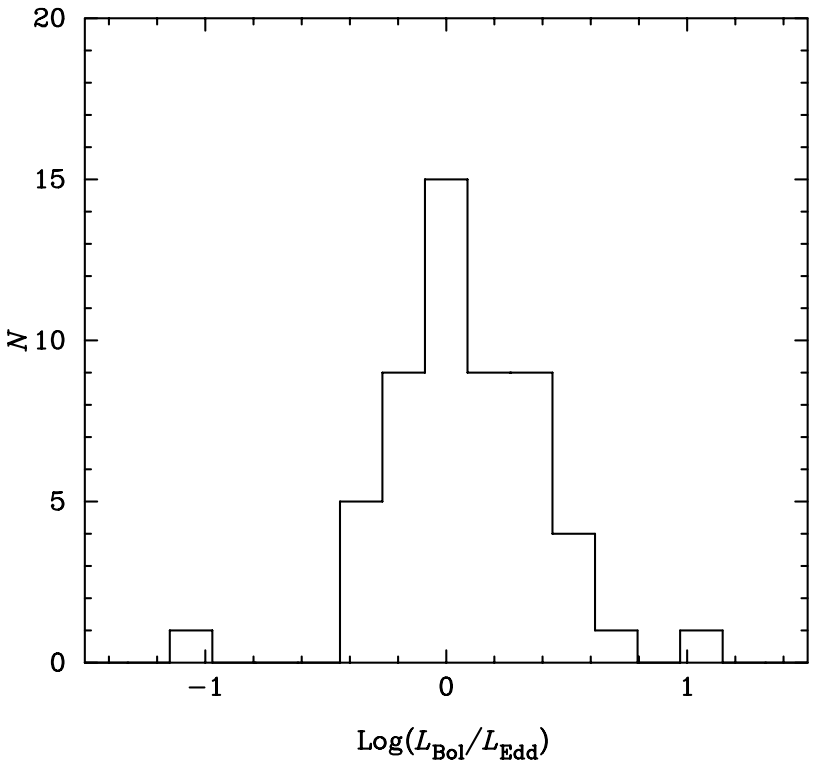

Fig. 4. The Eddington ratio distribution in the Véron-Cetty et al. (2001) sample calculated as explained in the text.

obtained by assuming a "typical" optical continuum of the form $L_{v} \propto v^{-0.5}$ and (as in Kaspi et al. 2000) a bolometric luminosity of $L_{\mathrm{Bol}} \approx 9 \lambda L_{\lambda}(5100 \AA)$. Given the measured $F W H M$, which is taken to be the one-dimensional gas velocity, we calculated $\mathrm{BH}$ masses assuming virialized BLR. Combining with the observed flux and redshift, we estimated the Eddington ratio for all 54 sources. The results are shown in Fig. 4.

Inspection of Fig. 4 shows that the mean of the sample is

$\left\langle\log \left(\frac{L_{\text {Bol }}}{L_{\text {Edd }}}\right)\right\rangle=0.08 \pm 0.31$,

i.e., most of the NLS1 are super-Eddington accretors (the two extreme cases are IRAS $05262+44$ and NGC 4051). Moreover, the theoretical results presented earlier, as well as the results of Wang \& Zhou (1999) and Ohsuga et al. (2002), suggest that in such sources the radiated luminosity is significantly lower than the accreted gravitational energy because of photon trapping. In particular, in PTAFs, $\dot{m} \gg L_{\mathrm{Bol}} / L_{\mathrm{Edd}}$. It thus seems reasonable to assume that most NLS1s are super-Eddington accretors.

As shown in the previous section, super-Eddington accretion can be identified by the presence of a soft X-ray hump in the spectrum. The following section addresses the possible observation of such features.

\subsection{Soft $X$-ray humps in NLS1s}

Soft X-ray humps in NLS1 spectra have been discussed in earlier papers (Turner et al. 1998; Leighly 1999; Vaughan et al. 1999; Comastri et al. 2001; Turner et al. 2001; Puchnarewicz et al. 2001; Collinge et al. 2001; O'Brien et al. 2001; Boller et al. 2001). There were several suggestions relating such features to blends of X-ray emission lines. We consider those unlikely due to theoretical reasons (Turner et al. 1999) as well as the lack of direct evidence in recent high resolution XMM observations of some of the sources. Below we discuss the spectrum of RE J1034+396 as an extreme case and address the possible connection to the theoretical spectra shown in the previous sections.

The ultra-soft X-ray radiation of RE J1034+396 ( $z=$ 0.003 ) was first discovered by Puchnarewicz et al. (1995). The spectrum shown in Fig. 5 shows a clear soft X-ray hump extending from $\log v=16.5$ (the lowest observed frequency) to 17.2. The data were taken from Puchnarewicz et al. (2001).

The fittings made by Puchnarewicz et al. (2001) to the spectrum of RE J1034+396 were based on the standard accretion disk model (Czerny \& Elvis 1987). The resulting accretion rate is $0.3-0.7 \dot{M}_{\text {cr }}$ and the inclination $\theta_{\text {obs }}=60-75^{\circ}$. Such a high accretion rate is inconsistent with a thin disk model. For a standard accretion disk model, the maximum frequency of the spectrum is roughly proportional to $\dot{m}^{1 / 4}$ and the luminosity is proportional to $\dot{m}$. A strong soft X-ray hump in such disks requires very large $\dot{m}$. This would give very large luminosity which is the reason why Puchnarewicz et al. (2001) had to assume high inclination (to decreases the total observed luminosity by a factor of $\cos \theta_{\text {obs }}$ ). Even with such extreme values of $\dot{m}$, there were difficulties fitting the observed spectrum.

The ESD model provides a natural way to increase $\dot{m}$ without a proportional increase in the bolometric luminosity. To illustrate this, we assume a face on system with $\alpha=\alpha_{\mathrm{c}}=0.1$. The mass of the central black hole can be obtained from equation (29). Figure 5 shows three such models fitted to the spectrum of RE J1034+396 representing $M_{\mathrm{BH}}=2.25 \times 10^{6} M_{\odot}$, $\dot{m}=5,10,20$ and $f=1.1 \times 10^{-2}, 5.5 \times 10^{-3}, 2.75 \times 10^{-3}$. The maximum frequency of the hump is determined by $r_{\text {in }}$ which, in this model, is fixed at $r_{\text {in }}=3$. The minimum frequency is determined by the accretion rate, as seen from the theoretical spectrum in Fig. 5. However, in this case the low frequency part of the spectrum is not observed and thus the accretion rate is unknown. There are only two parameters, $M_{\mathrm{BH}}$ and $r_{\mathrm{in}}$, that determine the total flux in the hump. The hard X-ray spectrum (at frequencies larger than $v_{\max }$ ) is determined by $f \dot{M}$ and $\alpha_{\mathrm{c}}$. Since $\dot{M}$ is unknown, $f$ is the real free parameter of the fit.

We find that $\dot{m}=5$ cannot provide a satisfactory fit to the data. The combination of $\dot{m}=10$ and $f \dot{m}=0.055$ provides a good fit for the spectrum of and $\dot{m}=20$ is also consistent with the data given the lack of observations at low X-ray energies. The large observational uncertainties on the hard X-ray spectrum translate to large uncertainty on the value of $f$. The best fit is $M_{\mathrm{BH}}=2.25 \times 10^{6} M_{\odot}, f \dot{M}=0.055 \dot{M}_{\mathrm{cr}}, \dot{M}=10 \dot{M}_{\mathrm{cr}}$ and $r_{\text {in }}=3.0$. We have also computed a model with $r_{\text {in }}=2.0$ and found very little change thus the dependence on $r_{\text {in }}$ over the fitted energy range is very weak.

The BH mass obtained from the model can be compared with the mass obtained by using the Kaspi et al. (2000) relationship for $R_{\mathrm{BLR}}$ vs. $L_{5100}$, and the observed line widths. Using the data in Puchnarewicz et al. (1995) with $F W H M(\mathrm{H} \beta)=$ $1500 \mathrm{~km} \mathrm{~s}^{-1}$, we get $M=4.1 \times 10^{6} M_{\odot}$, in good agreement with the spectra-fit results.

The present model allows two different kinds of X-ray variations. The hard X-ray spectrum is the Comptonized component of the soft hump flux. This means that intrinsic variations in the hump luminosity must result in correlated hard X-ray variations but not necessarily in optical-UV variations. On the other hand, the hard X-ray luminosity is very sensitive to the 


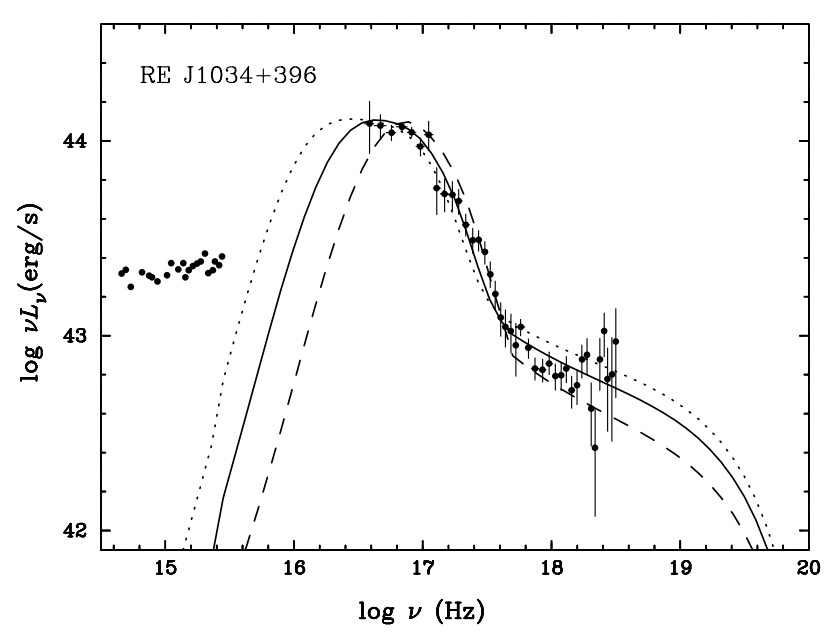

Fig. 5. The soft hump and hard X-ray spectrum of RE J1034+396. The data are taken from Puchnarewicz et al. (2001). The dashed, solid and dotted lines represent ESD models with $\dot{m}=5,10$, and 20 respectively, and $f \dot{m}=0.03$. In all cases $r_{\text {in }}=3$ and $M_{\mathrm{BH}}=2.25 \times 10^{6} M_{\odot}$. The fits include Comptonization in both the ESD and the hot corona.

value of $f$. Time dependent variations in $f$ will lead to variations in the hard X-ray flux yet the soft hump canremain constant because of photon trapping effects. This can be tested observationally.

\section{Discussion and conclusions}

In this paper we explored the structure and the spectrum of extreme slim disks with hot coronae. We focused on the question of whether such systems can fit, simultaneously, the soft X-ray hump and the hard X-ray spectrum of NLS1s. We presented a self-similar solution of ESDs with hot coronae and showed that their spectrum contains low energy humps produced by the ESD and a higher energy power-law continuum with a cutoff produced by the hot corona. We find that the structure of the hot corona, which we assumed to be Thomson-thin, is independent of the mass of the black hole and is fully specified by the parameters $f \dot{m}$ and $\alpha_{\mathrm{c}}$. The ESD structure and spectrum (the "hump") is insensitive to the accretion rate, but sensitive to the mass of the black hole. This can explain the similarity of the hard X-ray spectrum of Galactic black hole candidates and NLS1s despite of the very large difference in BH mass. Our present models apply to $\dot{m} \gg 2.5$ and $f<0.0375(\dot{m} / 10)^{-1}$. The calculations do not include GR effect and apply only to non-rotating BHs.

Slim disk fluctuations have been suggested by Mineshige et al. (2000) as the origin of the soft X-ray variations in such systems. The reason is the unusual energy balace

$u_{\mathrm{mag}} \sim u_{\mathrm{grav}}>u_{\mathrm{rad}}$

where $u$ denotes the energy density. The trapped photon accretion flows discussed in this work are likely to show two different types of variations: 1) simultaneous variations of the soft hump and the hard X-ray without any significant time lag; 2) small variability of the soft hump and larger variation in the hard X-ray spectrum. The first type is related to the so called "photon bubble instability" (Gammie 1999) that can be very important in SDs since the energy densities of the trapped photons and the magnetic field are larger than those in standard accretion disks. In this case, the hard X-rays will follow closely the soft hump variations. Using the simplest version of Comptonization (Sunyaev \& Titarchuk 1980) we find that the ratio of the hard X-ray flux to the soft hump flux is roughly $p(p+3)(2 p+3)^{-1}\left(v_{\mathrm{HX}} / v_{\text {hump }}\right)^{-p}$ where $v_{\mathrm{HX}}$ and $v_{\text {hump }}$ are representative frequencies of the hard and the soft (hump) X-ray flux. This ratio is sensitive to the hard X-ray spectrap index $p$. This type of variations agrees with at least some observations (e.g. Leighly 1999; Dewangan et al. 2002). Regarding the second type, here the fluctuations are due to changes in the accretion rate. Such fluctuations cause large hard X-ray variations since that radiation is sensitive to the accretion. On the other hand, the soft hump flux remains almost constant due to photon trapping. This type of variations has been reported in the Chandra observations of $1 \mathrm{H}$ 0707-495 (Leighly et al. 2002). It will be interesting to apply a more detailed combined spectral and stability analysis of this type to the observed X-ray spectrum of NLS1s.

The calculations presented here include the new ingredients of a hot corona, yet they are still oversimplified in various ways. First, we assumed a continuous corona which is characterized by a single parameter $f$. However, patchy corona may be more reaslistic for SDs. Second, the hot corona gas is assumed to rotate with Keplerian velocity. This leads to an underestimate of the advection in the hot corona since the hot gas may be sub-Keplerian. This uncertainty can be aborbed into the factor $f$. A possible improvement is to use the optically thin advection-dominated self-similar solution (Narayan \& Yi 1994) as an approximation of the hot corona. We also note that for the case of standard accretion disk, the factor $f$ approaches unity (Svensson \& Zdzarski 1994). However, in that case feedback to the cold disk can be neglectable since $f \ll 1$ in the super-Eddington case. Third, we do not take into account the irradiation of the disk due to the hot corona. Future work will have to treat the more general case of irradiation and try to solve, self-consistently, for the disk-corona interface e.g. via ionization instability (Życki et al. 2000).

Another limitation of the model is the neglect of GR effects. Thus, the treatment of the physical processes near the disk inner boundary, and the calculation of the emitted spectrum from this region, are not very accurate. As explained, global energy considerations show that the models presented here do not deviate much from the more realistic cases since a large fraction of the radiation is emitted way outside of $R_{\text {in }}=3$, our standard inner radius. In general we expect models with full GR treatment and similar $\dot{m}$ to show a very similar hump and to require a somewhat smaller $f$ for the same hard X-ray flux. We will test these qualitative estimates in a future paper. Finally, we neglected any spectral dependence on the inclination angle of the disk. This will be included in future models.

Our new model enables a comparison with several already observed NLS1s. Study of the Véron-Cetty et al. (2001) sample showed that the mean Eddington ratio in this sample is larger than unity which suggests super-Eddington accretion rates in 
many NLS1. As an application of the model, we fitted the spectrum of RE J1034+396 and obtained a good agreement, within the observational uncertainties, over the entire observed X-ray band. We also find that the mass of the central black hole obtained from the model is in good agreement with the mass obtained by using the reverberation mapping method.

Regarding new observations to test the model, perhaps the most important are Chandra and XMM spectra that cover the lowest energy range. This will enable a careful study of the "red" side of the ESD hump in several sources and a more direct determination of the accretion rate. A careful study of the "knee" spectral region is another observational challenge with implications to the type of black hole (via the value of $R_{\text {in }}$ ) and the energy production in the corona (via the value of $f$ ).

Acknowledgements. We are grateful to the referee for the useful comments and suggestions that helped improve this paper. We also thank A. Laor for his careful reading of an early version of the manuscript and for providing useful comments. We acknowledge useful discussions with O. Shemmer, D. Chelouche and S. Mineshige. This research was supported by the Israel Science Foundation (grant no. 545/00) and by the Special Funds for Major State Basic Research Projects and NSFC. JMW is supported by Hundred Talents Program of Chinese Academy of Sciences.

\section{References}

Abramowicz, M. A., Czerny, B., Lasota, J. P., \& Szuszkiewicz, E. 1988, ApJ, 332, 646

Begelman, M. C., \& Meier, D. L. 1982, ApJ, 253, 873

Blandford, R. 1990, in Active Galactic Nuclei, ed. R. Blandford, H. Netzer, \& L. Woltjer (Springer), 209

Boller, T., Brandt, W. N., \& Fink, H. 1996, A\&A, 305, 53

Boller, T., Keil, R., Trümper, J., et al. 2001, A\&A, 365, L146

Boller, T., Fabian, A. C., Sunyaev, R., et al. 2002, MNRAS, 329, L1

Boroson, T., \& Green, R. F. 1992, ApJS, 80, 109

Brandt, N., \& Boller, Th. 1998, AN, 319, 163

Chakrabarti, S. K., \& Titarchuk, L. G. 1995, ApJ, 455, 623

Chen, X. 1995, MNRAS, 275, 641

Collier, S., Crenshaw, D. M., Peterson, B. M., et al. 2001, ApJ, 561, 146

Collin, S. 2001, Lectures given at GH Advanced Lectures on the Starburst-AGN Connection, INAOE, June 2000, ed. D. Kunth, \& I. Aretxaga [astro-ph/0101203]

Collin, S., Boisson, C., Mouchet, M., et al. 2002, A\&A, 388, 771

Collin, S., \& Huré, J.-M. 2001, A\&A, 372, 50

Collinge, M. J., Brandt, W. N., Kaspi, Shai, et al. 2001, ApJ, 557, 2

Comastri, A., Stirpe, G. M., Vignali, C., et al. 2001, A\&A, 365, 400

Czerny, B., \& Elvis, M. 1987, ApJ, 321, 305

Dewangan, G. C., Boller, Th., Singh, K. P., \& Leighly, K. M. 2002, A\&A, 390, 65

Dörrer, T., Riffert, H., Staubert, R., \& Ruder, H. 1996, A\&A, 311, 69

Fiore, F., Matt, G., Cappi, M., et al. 1998, MNRAS, 298, 103

Fukue, J. 2000, PASJ, 52, 105

Gammie, C. F. 1998, MNRAS, 297, 929

George, I. M., Turner, T. J., Yaqoob, T., et al. 2000, ApJ, 531, 52

Gliozzi, M., Brinkmann, W., Laurent-Muehleisen, S. A., Moran, E. C., \& Whalen, J. 2001, A\&A, 377, 44

Haardt, F., \& Maraschi, L. 1991, ApJ, 380, L51

Hubeny, I., \& Hubeny, V. 1997, ApJ, 484, L37
Hua, X., \& Titarchuk, L. 1995, ApJ, 449, 188

Janiuk, A., \& Czerny, B. 2000, New Astron., 5, 7

Kaspi, S., Smith, P., Netzer, H., et al. 2000, ApJ, 533, 631

Kawaguchi, T., Shimura, T., \& Mineshige, M. 2001, ApJ, 546, 966

Krolik, J. H., \& Hawley, J. F. 2002, ApJ, 573, 754

Laor, A. 1990, MNRAS, 246, 369

Laor, A., Fiore, F., Elvis, M., Wilkes, B. J., \& McDowell, J. C. 1994, ApJ, 435, 611

Laor, A., Fiore, F., Elvis, M., Wilkes, B. J., \& McDowell, J. C. 1997, ApJ, 477, 93

Laor, A., \& Netzer, H. 1989, MNRAS, 238, 897

Leighly, K. M. 1999, ApJS, 125, 317

Leighly, K. M., et al. 2002 [astro-ph/0205539]

Liang, E. P. T., \& Price, R. H. 1977, ApJ, 218, 247

Malkan, M. A., \& Sargent, W. L. W. 1982, ApJ, 254, 22

Malkan, M. A. 1983, ApJ, 268, 582

Matsumoto, R., Kato, S., Fukue, J., \& Okazaki, A. T. 1984, PASJ, 36 , 71

Mineshige, S., Takeuchi, M., \& Nishimori, H. 1994, ApJ, 435, L125

Mineshige, S., Kawaguchi, T., Takeuchi, M., \& Hayashida, K. 2000, PASJ, 52, 499

Muchotrzeb, B., \& Paczyński, B. 1982, Acta Astr., 32, 1

O'Brien, P. T., Reeves, J. N., Turner, M. J. L., et al. 2001, A\&A, 365, L122

Ohsuga, K., Mineshige, S., Mori, M., \& Umemura, M. 2002, ApJ, 574,315

Peterson, B. M., McHardy, I. M., Wilkes, B. J., et al. 2000, ApJ, 542, 161

Pounds, K. A., Done, C., \& Osborne 1995, MNRAS, 277, L5

Puchnarewicz, E. M., Mason, K. O., Siemiginowska, A., \& Pounds, K. A. 1995, MNRAS, 276, 20

Puchnarewicz, E. M., Mason, K. O., Siemiginowska, A., et al. 2001, ApJ, 550, 644

Ross, R., Fabian, A. C., \& Mineshige, S. 1992, MNRAS, 258, 189

Shakura, N. I., \& Sunyeav, R. A. 1973, A\&A, 24, 337

Shapiro, S. L., Lightman, A. P., \& Eardley, D. M. 1976, ApJ, 204, 187

Shemmer, O., Romano, P., Bertam, R., et al. 2001, ApJ, 561, 162

Shields, G. A. 1978, Nature, 272, 706

Shimura, T., \& Takahara, F. 1993, ApJ, 419, 78

Sincell, M. W., \& Krolik, J. H. 1997, ApJ, 476, 605

Spruit, H. C. 2000 [astro-ph/0003143]

Störzer, H., Hauschildt, P. H., \& Allard, F. 1994, ApJ, 437, L91

Sun, W.-H., \& Malkan, M. A. 1989, ApJ, 346, 68

Sunyaev, R., \& Titarchuk, L. 1980, A\&A, 86, 121

Svensson, R., \& Zdzarski, A. 1994, ApJ, 436, 599

Szuszkiewicz, E., Abramowicz, M., \& Malkan, M. A. 1996, ApJ, 458, 474

Turner, T. J., George, I. M., Yaqoob, T., et al. 2001a, ApJ, 548, L13

Turner, T. J., Romano, P., George, I. M., et al. 2001b, ApJ, 561, 131

Turner, T. J., Romano, P., Kraemer, S. B., et al. 2002, ApJ, 568, 120

Turner, T. J., George, I. M., \& Nandra, K. 1998, ApJ, 509, 648

Turner, T. J., George, I. M., \& Netzer, H. 1999, ApJ, 526, 524

Wandel, A., \& Petrosian, V. 1988, ApJ, 319, L11

Wang, J.-M. 1999, Chin. Phys. Lett., 16, 467

Wang, J.-M., Szuszkiewicz, E., Lu, F. J., \& Zhou, Y. Y. 1999, ApJ, 522,839

Wang, J.-M., \& Zhou, Y.-Y. 1999, ApJ, 516, 420

Wang, T. G., \& Lu, Y.-J. 2001, A\&A, 377, 52

Watarai, K.-Y., \& Mineshige, S. 2001, PASJ, 53, 915

Vaughan, S., Reeves, J., Warwick, R., \& Edelson, R. 1999, MNRAS, 309, 113

Véron-Cetty, M.-P., Véron, P., \& Goncalves, A. C. 2001, A\&A, 372, 730

Życki, P. T., Collin-Souffrin, S., \& Czerny, B. 1995, MNRAS, 277, 70 